\title{
Loss of ATF3 exacerbates liver damage through the activation of mTOR/p70S6K/ HIF-1a signaling pathway in liver inflammatory injury
}

Qiang Zhu ${ }^{1,2}$, Han Wang ${ }^{1}$, Bin Jiang ${ }^{2}$, Xuhao Ni ${ }^{1}$, Longfeng Jiang ${ }^{1}$, Changyong $\mathrm{Li}^{3}$, Xuehao Wang ${ }^{1}$, Feng Zhang ${ }^{1}$, Bibo $\mathrm{Ke}^{4}$ and Ling $\mathrm{Lu}^{1}$

\begin{abstract}
Activating transcription factor 3 (ATF3) is a stress-induced transcription factor that plays important roles in regulating immune and metabolic homeostasis. Activation of the mechanistic target of rapamycin (mTOR) and hypoxia-inducible factor (HIF) transcription factors are crucial for the regulation of immune cell function. Here, we investigated the mechanism by which the ATF3/mTOR/HIF-1 axis regulates immune responses in a liver ischemia/reperfusion injury (IRI) model. Deletion of ATF3 exacerbated liver damage, as evidenced by increased levels of serum ALT, intrahepatic macrophage/neutrophil trafficking, hepatocellular apoptosis, and the upregulation of pro-inflammatory mediators. ATF3 deficiency promoted mTOR and p70S6K phosphorylation, activated high mobility group box 1 (HMGB1) and TLR4, inhibited prolyl-hydroxylase 1 (PHD1), and increased HIF-1a activity, leading to Foxp3 downregulation and RORyt and IL-17A upregulation in IRI livers. Blocking mTOR or p70S6K in ATF3 knockout (KO) mice or bone marrow-derived macrophages (BMMs) downregulated HMGB1, TLR4, and HIF-1a and upregulated PHD1, increasing Foxp3 and decreasing IL-17A levels in vitro. Silencing of HIF-1a in ATF3 KO mice ameliorated IRI-induced liver damage in parallel with the downregulation of IL-17A in ATF3-deficient mice. These findings demonstrated that ATF3 deficiency activated mTOR/p70S6K/HIF-1a signaling, which was crucial for the modulation of TLR4-driven inflammatory responses and T cell development. The present study provides potential therapeutic targets for the treatment of liver IRI followed by liver transplantation.
\end{abstract}

\section{Introduction}

Liver ischemia and reperfusion injury (IRI) is a major problem associated with liver transplantation and resection. Liver inflammatory responses induced by IR can exacerbate liver damage. Macrophages (Kupffer cells) play a critical role in triggering TLR4-mediated innate immune

\footnotetext{
Correspondence: Bibo Ke (bke@mednet.ucla.edu) or

Ling. Lu (Ivling@njmu.edu.cn)

${ }^{1}$ Liver Transplantation Center, First Affiliated Hospital, Nanjing Medical

University, Nanjing, China

${ }^{2}$ Children's Hospital of Nanjing Medical University, Nanjing, China

Full list of author information is available at the end of the article.

These authors contributed equally: Qiang Zhu, Han Wang, Bin Jiang

Edited by T. Kaufmann
}

responses and in liver inflammation ${ }^{1,2}$. IR-induced liver inflammation leads to the release of endogenous damageassociated molecular pattern (DAMP) molecules, which activate the TLR4 signaling cascade on Kupffer cells and the release of pro-inflammatory cytokines leading to the activation of $\mathrm{T}$ cells ${ }^{3-5}$. Recent studies showed that $\mathrm{T}$ cells can differentiate into IL-17-producing cells, a distinct $\mathrm{CD}^{+}{ }^{+} \mathrm{T}$ cell lineage that is independent from $\mathrm{Th} 1$ or Th2 cell development ${ }^{6,7}$. Th17 cells contribute to the inflammatory response by mediating the recruitment of macrophages and neutrophils to injured tissues ${ }^{7}$. Moreover, RORyt-expressing $\left(\mathrm{ROR}_{\mathrm{t}} \mathrm{t}^{+}\right) \mathrm{T}$ cells are the main source

\section{(c) The Author(s) 2018}

(c) (i) Open Access This article is licensed under a Creative Commons Attribution 4.0 International License, which permits use, sharing, adaptation, distribution and reproduction c. in any medium or format, as long as you give appropriate credit to the original author(s) and the source, provide a link to the Creative Commons license, and indicate if changes were made. The images or other third party material in this article are included in the article's Creative Commons license, unless indicated otherwise in a credit line to the material. If material is not included in the article's Creative Commons license and your intended use is not permitted by statutory regulation or exceeds the permitted use, you will need to obtain permission directly from the copyright holder. To view a copy of this license, visit http://creativecommons.org/licenses/by/4.0/. 
of Th17-producing cells during the early phase of liver $\mathrm{IRI}^{8}$. We previously demonstrated that RORyt/IL-17A ${ }^{+}$expressing $\mathrm{T}$ cells played a crucial role in mediating hepatic IRI ${ }^{9}$.

Activating transcription factor 3 (ATF3), a basic leucine zipper (bZIP) DNA binding protein, is a member of the ATF/cAMP responsive element binding protein (CREB) family of transcription factors. Under normal conditions, ATF3 is expressed at minimal levels. However, ATF3 can be induced by various stress signals including ischemia ${ }^{10}$, ER stress ${ }^{11}$, endotoxins, and cytokines $^{12}$. ATF3 is rapidly and preferentially induced during the early stage of the inflammatory response in organ IRI, such as in the kidney ${ }^{13,14}$ and brain ${ }^{15}$. Overexpression of ATF3 inhibits oxidative stress-induced apoptotic cell death in renal cells ${ }^{13}$, whereas disruption of ATF3 increases pro-inflammatory cytokine release, leading to increased susceptibility to endotoxic shockinduced cell death ${ }^{16}$.

The mechanistic target of rapamycin (mTOR) forms two distinct multi-protein complexes, mTOR complex1 (mTORC1) and mTOR complex2 (mTORC2) ${ }^{17}$. As anatypical serine/threonine kinase, mTOR plays important roles in the regulation of metabolism, cell growth, and proliferation $^{18}$. Constitutive mTORC1 activation in macrophages promotes $\mathrm{M} 1$ and impairs alternative M2 polarization to enhance the inflammatory response in vitro and in vivo ${ }^{19,20}$. Inhibition of mTORC1 reduces LPS-induced pro-inflammatory cytokine production by suppressing NF- $\mathrm{kB}$ activation in macrophages ${ }^{21}$. Moreover, increased mTOR activity promotes $\mathrm{T}$ helper (Th) cell responses by reprograming metabolic processes ${ }^{22}$. Loss of mTOR results in failure of effector $\mathrm{CD} 4^{+} \mathrm{T}$ cell differentiation, whereas it induces forkhead box protein 3

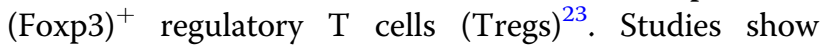
that mTOR is a key regulatory factor for Th17 differentiation ${ }^{24}$.

Hypoxia-inducible factors (HIFs) are transcription factors that respond to low oxygen concentration or hypoxia. HIF-1 is a basic helix-loop-helix-PAS heterodimer composed of an alpha and a beta subunit ${ }^{25}$. HIF-1 alpha subunit (HIF-1 $\alpha)$ is regulated by prolylhydroxylase domain (PHD) proteins. Under normoxia, PHD enzymes catalyze the hydroxylation of two highly conserved proline residues within the oxygendependent degradation (ODD) domain of HIF-1 $\alpha$ by the E3 ubiquitin ligase von Hippel-Lindau (VHL)mediated ubiquitin-proteasome pathway ${ }^{26}$. However, reduced PHD activity results in rapid HIF- $\alpha$ accumulation, nuclear translocation, and activation of hypoxia targeting genes under hypoxic conditions ${ }^{26}$. HIF- $1 \alpha$ has a pivotal regulatory function in innate and adaptive immune cells. Disruption of myeloid-specific HIF- $1 \alpha$ inhibits inflammatory responses by impairing macrophage aggregation and invasion ${ }^{27}$. HIF- $1 \alpha$ deletion in $\mathrm{T}$ cells also reduces inflammatory responses by promoting Foxp $3^{+}$Treg and inhibiting TH17 cell differentiation $^{28}$. Moreover, the absence of the mTOR signaling motif diminishes HIF- $1 \alpha$ activity during hypoxia ${ }^{29}$, implying a mechanistic link between mTOR signaling and HIF-1 $\alpha$ activity during hypoxia. Despite the known role of ATF3 in controlling innate inflammatory responses and the involvement of HIF- $1 \alpha$ in mTOR signaling, the exact mechanisms by which ATF3 regulates innate immunity and adaptive $\mathrm{T}$ cell development in IR-triggered liver inflammation remain largely unknown.

In the present study, we showed that ATF3 deficiency aggravated IR-induced liver inflammation by activating of mTOR/p70S6K signaling and increasing TLR4-driven inflammatory responses. Activation of mTOR upregulated HIF-1 $\alpha$, whereas inhibiting PHD1 activity reduced Foxp $3^{+}$Tregs and promoted Th17 cell differentiation in IR-induced liver injury. These data indicated that ATF3mediated mTOR/p70S6K//HIF-1 $\alpha$ signaling is a novel regulator of innate and adaptive immunity in IR-induced liver injury.

\section{Results}

\section{ATF3 deficiency exacerbates hepatocellular damage in IR-induced liver injury}

To determine the effects of ATF3 in different cells on liver IRI, the expression of ATF3 was detected in hepatocytes and infiltrating macrophages at various time points after reperfusion (Fig. 1a and Supplemental Figure 1). Then, hepatocellular function was assessed in mouse livers subjected to $90 \mathrm{~min}$ of warm ischemia followed by $6 \mathrm{~h}$ of reperfusion ${ }^{30}$. The livers of ATF3 KO mice showed severe edema, sinusoidal congestion, and necrosis (Fig. 1a, b, score $=2.98 \pm 0.35$ ). In contrast, the livers of WT mice showed mild to moderate edema and sinusoidal congestion (Fig. 1a, b, score $=1.3 \pm 0.34$, $p<0.001)$. The levels of serum ALT (IU/L) were significantly higher in ATF3 KO mice than in the WT controls (Fig. 1c, $9736 \pm 973$ vs. $4634 \pm 603, p<0.001$ ). The results of MPO assay, showed that hepatic neutrophil activity $(\mathrm{U} / \mathrm{g})$, was $3.2 \pm 0.27$ in the WT and $6.45 \pm 1.32$ in the ATF3 KO group (Fig. $1 \mathrm{~d}, p=0.004$ ). Consistent with these data, ATF3 KO increased the frequency of $\mathrm{TUNEL}^{+}$cells in ischemic livers compared with that in the WT controls (Fig. 1e, f, $80.4 \pm 5.68$ vs. $39.2 \pm 2.28 ; p<0.001)$. Unlike the WT controls, the protein expression of anti-apoptotic proteins $(\mathrm{Bcl}-2$ and BCL-xL) was decreased in ATF3 KO livers (Fig. 1g). This was confirmed by increased caspase- 3 activity in ATF3 KO but not in WT controls (Fig. 1h). These results indicated that knockdown of ATF3 exacerbated IR-induced liver damage. 


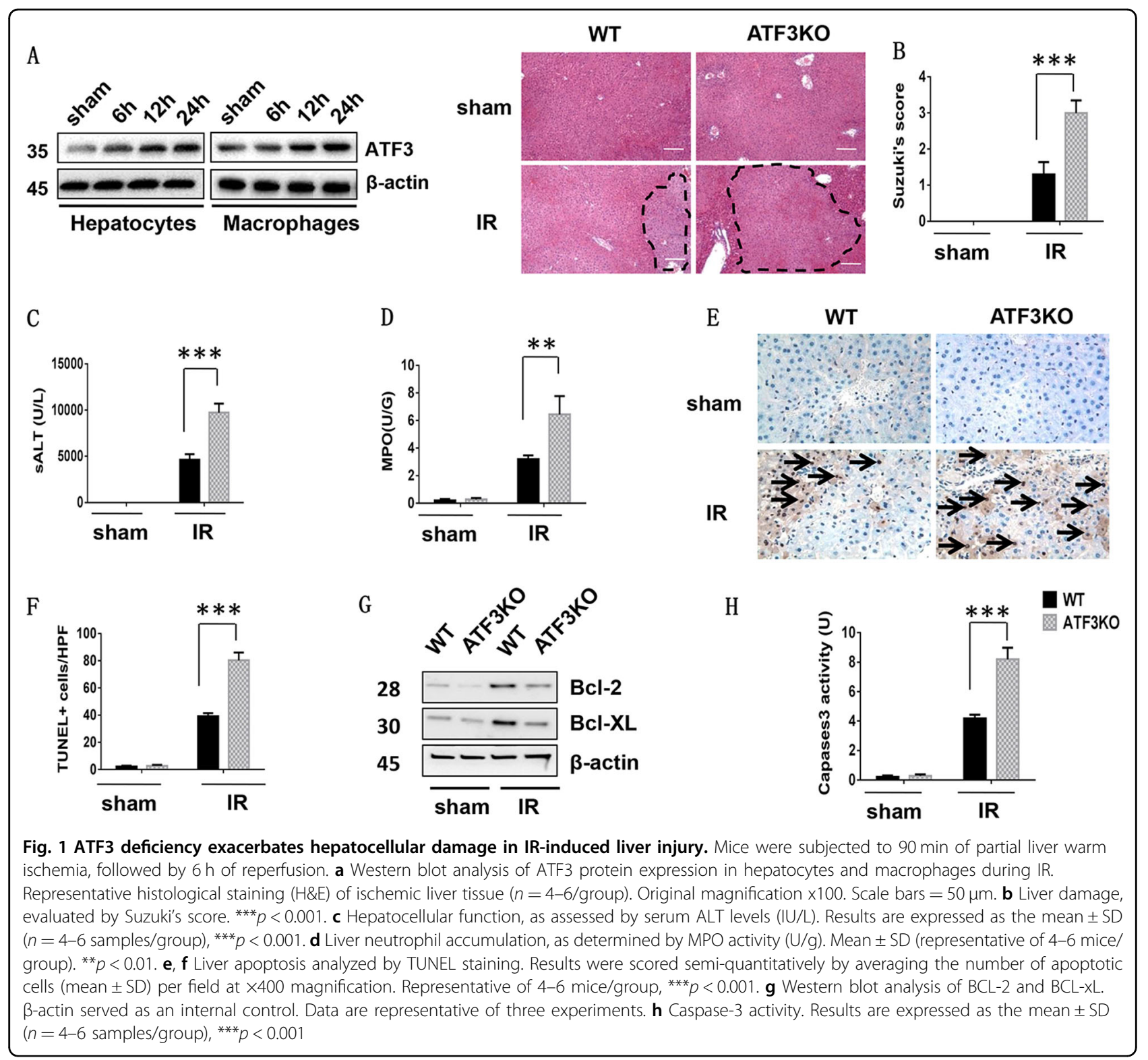

ATF3 deficiency increases macrophage/neutrophil trafficking, promotes mTOR and TLR4 activation, and induces HIF-1a signaling and $\mathrm{T}$ cell differentiation in IR-induced liver injury

To determine whether ATF3 affected inflammatory cell recruitment in ischemic livers, $\mathrm{CD}_{11} \mathrm{~b}^{+}$macrophages and ${\text { Ly } 6 G^{+}}^{+}$neutrophils were detected by immunohistochemistry. $\mathrm{CD}_{11 \mathrm{~b}^{+}}$macrophages and $\mathrm{Ly}_{6 \mathrm{G}}{ }^{+}$neutrophils were increased in ATF3 KO but not in WT mice (Fig. 2a, $41 \pm$ 3.53 vs. $19.4 \pm 1.67, p<0.001 ; 49.4 \pm 4.56$ vs. $23.8 \pm 3.03$, $p<0.001$, respectively). ATF3 KO upregulated TNF- $\alpha$, IL- $1 \beta$, and IL- 6 and downregulated TGF- $\beta$ expression in ischemic livers compared with the WT controls (Fig. 2b). The protein expression of phospho-mTOR, phosphop70S6K, and TLR4 was upregulated in parallel with PHD1 downregulation and HIF- $\alpha$ upregulation in ATF3 KO livers compared with WT livers (Fig. 2c). In addition, ATF3 KO significantly reduced the percentage of splenic CD ${ }^{+}{ }^{+}$D $25^{+}$Foxp $^{+}$Tregs (Fig. 2 d, $8.8 \pm 1.18$ vs. $13.86 \pm$ 1.42, $p<0.001)$ and increased CD $4^{+}$RoR $^{+}{ }^{+}$TH17 cells (Fig. $2 \mathrm{e}, 8.75 \pm 0.77$ vs. $3.59 \pm 0.41, p<0.001$ ), and this was accompanied by increased serum levels of IL-17A (Fig. 2f, $101.75 \pm 16.8$ vs. $45 \pm 6.05, p=0.003$ ) compared with the WT controls. Finally, F4/80 and CD11b double-positive macrophages were isolated from normal (sham) and IR livers. Western blot analysis showed that the protein expression of phospho-mTOR and phospho-p70S6K in macrophages was higher in ATF3 KO than in WT livers (Fig. 2g, h). These results suggested that ATF3 played an important role in the regulation of innate TLR4 


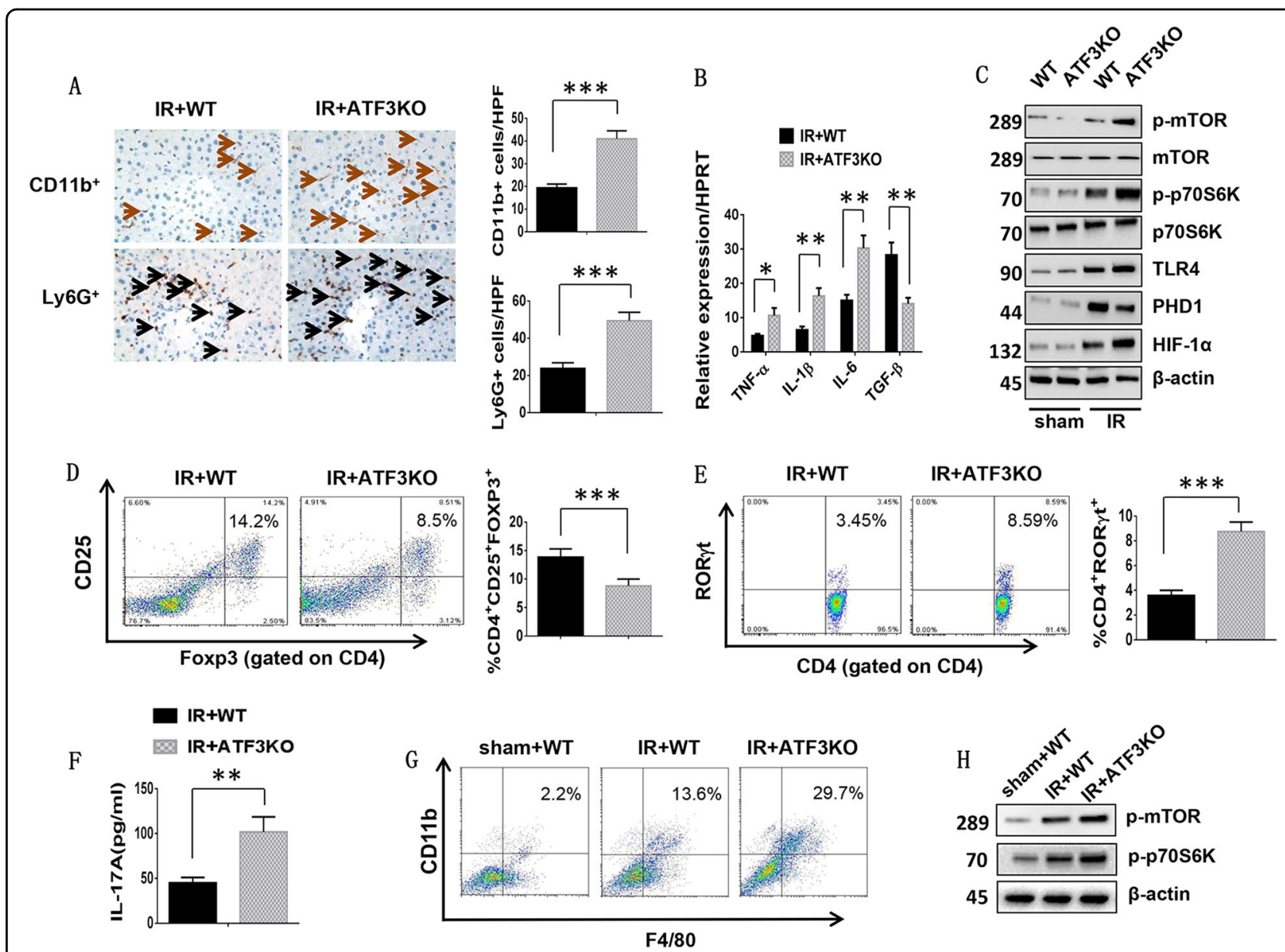

Fig. 2 ATF3 deficiency increases macrophage/neutrophil trafficking, promotes mTOR and TLR4 activation, induces HIF-1a signaling and T cell differentiation in IR-induced liver injury. a Liver $\mathrm{CD}_{11} \mathrm{~b}^{+}$macrophages and $\mathrm{Ly}_{6 \mathrm{G}}{ }^{+}$neutrophils were detected by immunohistochemistry. Results were scored semi-quantitatively by averaging the number of positively stained cells (mean \pm SD)/field at $\times 400$ magnification. Representative of 4-6 mice/group. ${ }^{* *} p<0.001$. b Quantitative RT-PCR-assisted detection of TNF- $a, \mathrm{IL}-1 \beta$, IL-6, and TGF- $\beta$ expression. Mean \pm SD ( $n=3-4$ samples/ group). ${ }^{*} p<0.05,{ }^{* *} p<0.01$. c Western blot analysis of phosphorylated mTOR, phosphorylated p70S6K, PHD1, HIF-1a, and TLR4. $\beta$-actin served as an internal control. Data are representative of three experiments. d, e Foxp3 and RORyt expression in spleen T cells was evaluated by flow cytometry. Representative of three separate experiments. ${ }^{* *} p<0.001$. f ELISA analysis of serum IL-17A levels. Mean \pm SD $\left(n=3-4\right.$ samples/group). ${ }^{* *} p<0.01$. g Cells were stained with fluorochrome-conjugated anti-F4/80 or -CD11b. F4/80 and CD11b double-positive cells were identified as infiltrating macrophages. $\mathbf{h}$ Western blot analysis of phosphorylated mTOR and phosphorylated p70S6K in infiltrating macrophages. $\beta$-actin served as an internal control. Data are representative of three experiments

and adaptive $\mathrm{T}$ cell differentiation during liver inflammatory injury.

Inhibition of mTOR signaling ameliorates ATF3 deficiencymediated liver damage in IR-induced liver injury

To test whether mTOR played a role in ATF3-mediated immune regulation during liver IRI, mTOR activity in ischemic livers was inhibitd by rapamycin. Pretreatment of ATF3 KO mice with rapamycin significantly improved edema, sinusoidal congestion/cytoplasmic vacuolization, and necrosis (Fig. 3a, b, $1.1 \pm 0.22$ vs. $3.2 \pm 0.27, p<0.001$ ), and decreased the frequency of TUNEL ${ }^{+}$cells (Fig. 3a, b, $40 \pm 2.54$ vs. $82.2 \pm 5.8, p<0.001)$ in ischemic livers compared with the DMSO vehicle controls. Consistent with the histological data, serum ALT levels (IU/L) were significantly lower in rapamycin-treated ATF3 KO mice than in the DMSO controls (Fig. 3c, $4852 \pm 536$ vs. $9786 \pm$ $1092, p<0.001)$. Moreover, rapamycin treatment in ATF3 $\mathrm{KO}$ mice reduced liver $\mathrm{CD}_{11 \mathrm{~b}^{+}}$macrophage (Fig. $3 \mathrm{~d}$, $21.2 \pm 2.16$ vs. $40.8 \pm 4.32, p<0.001)$ and $\mathrm{Ly} 6 \mathrm{G}^{+}$neutrophil recruitment (Fig. 3d, $24.5 \pm 0.29$ vs. $49.8 \pm 4.81$, $p<0.001)$ compared with the DMSO-treated controls. The protein expression of phospho-mTOR, phosphop70S6K, HMGB1, and TLR4 was decreased, whereas PHD1 was upregulated and HIF- $1 \alpha$ was downregulated (Fig. 3e), and this was accompanied by the downregulation of TNF- $\alpha$, IL- $1 \beta$, and IL- 6 and the upregulation of TGF- $\beta$ expression in rapamycin-treated livers (Fig. 3f) 


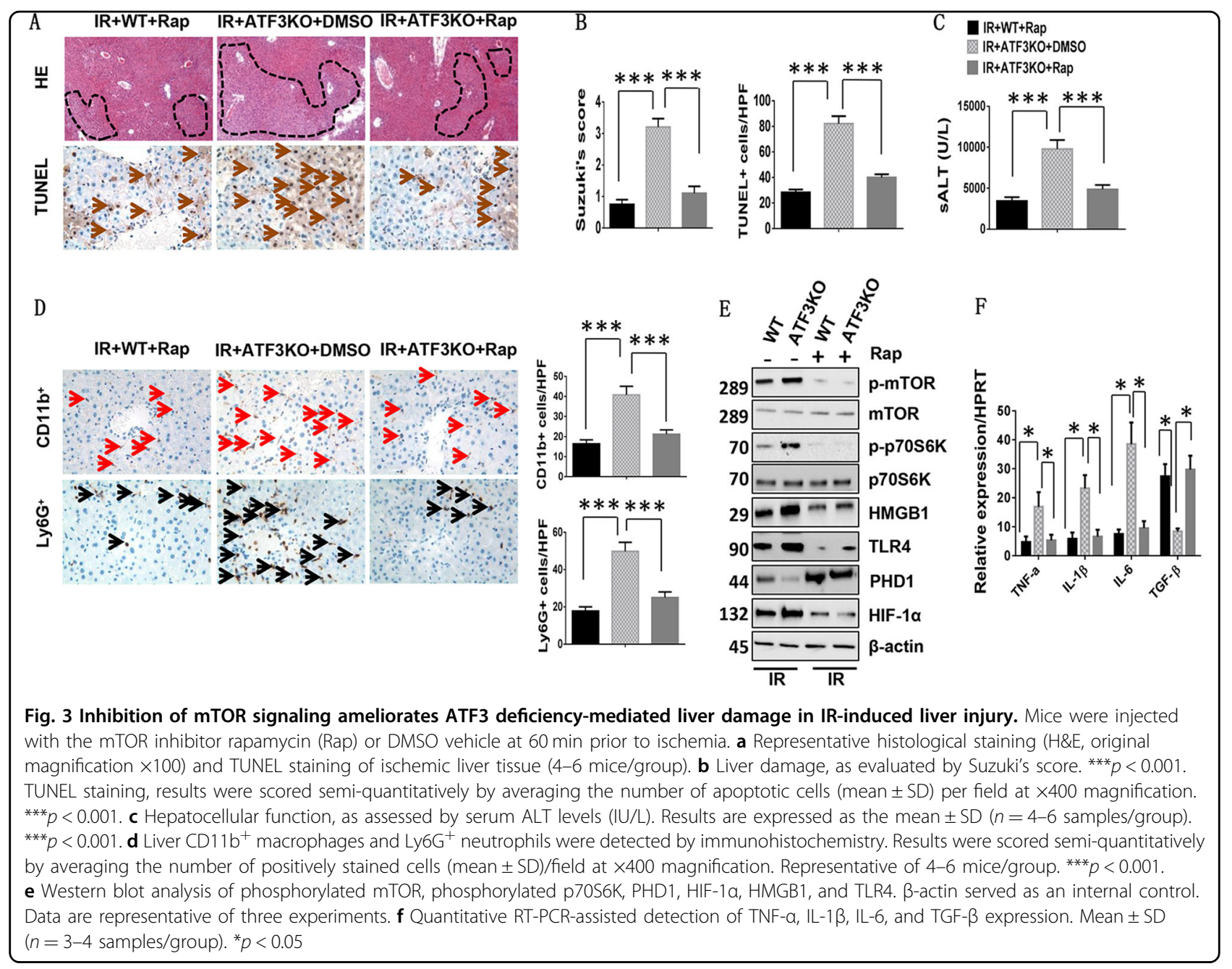

compared with the DMSO-treated controls. These findings suggested that mTOR was critical for ATF3mediated immune regulation during liver IRI.

\section{Blocking ATF3 deficiency-induced mTOR signaling inhibits TLR4 and HIF-1a signaling, promotes Foxp $3^{+}$Tregs, and inhibits Th17 cell differentiation in vitro}

To determine the mechanisms underlying the role of ATF3 deficiency-induced mTOR signaling in the regulation of innate TLR4, HIF-1 $\alpha$ signaling and adaptive T cells differentiation, mTOR activity was inhibited by rapamycin in BMMs from ATF3 KO mice. ATF3 KO upregulated phospho-mTOR and phospho-p70S6K, downregulated PHD1 and upregulated HIF- $1 \alpha$ in LPS-stimulated BMMs after DMSO treatment. However, inhibition of mTOR by rapamycin pretreatment downregulated phospho-mTOR, phospho-p70S6K, and HIF-1 $\alpha$ and upregulated PHD1 after LPS stimulation (Fig. 4a). Moreover, rapamycin treatment downregulated HMGB1 and TLR4 in ATF3deficient BMMs compared with the DMSO-treated controls (Fig. 4b). Decreased TNF- $\alpha$, IL-1 $\beta$, and IL-6 levels and increased TGF- $\beta$ mRNA levels were observed in rapamycin-treated cells but not in DMSO-treated controls after LPS stimulation (Fig. 4c). To determine whether macrophage ATF3 deficiency affected mTOR/ HIF- $1 \alpha$ signaling and $\mathrm{T}$ cell differentiation, rapamycinpretreated ATF3-deficient BMMs were co-cultured with spleen $\mathrm{CD}^{+}{ }^{+} \mathrm{T}$ cells after LPS stimulation. Inhibition of mTOR in ATF3-deficient BMMs decreased the mRNA levels of RORyt and IL-17A and increased Foxp3 levels in spleen $\mathrm{CD}^{+}{ }^{+} \mathrm{T}$ cells (Fig. 4d). Consistent with these data, ELISA-assessed IL-17A levels were significantly decreased in rapamycin-pretreated groups compared with those in DMSO-treated controls in co-culture supernatants (Fig. 4e). To further determine whether PHD1, HIF-1a, S6K, and mTOR were specifically modulated by ATF3, we used Ad-ATF3 or Ad-con to overexpress ATF3 in BMMs from WT mice. ATF3 overexpression inhibited the expression of phospho-mTOR, phosphop70S6K, upregulated PHD1, and downregulated HIF- $1 \alpha$ 


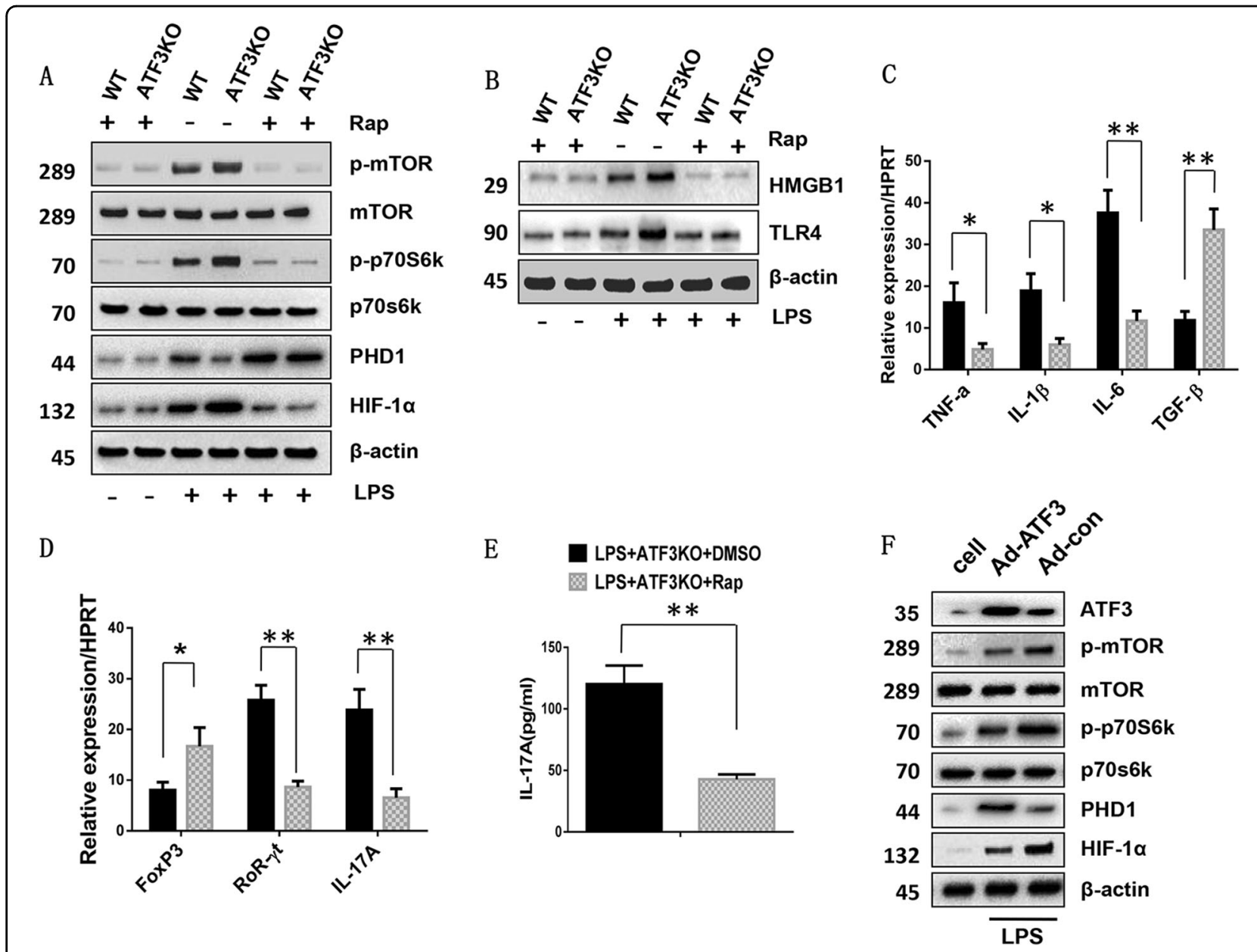

Fig. 4 Blocking ATF3 deficiency-induced mTOR signaling inhibits TLR4 and HIF-1a signaling, promotes Foxp $3^{+}$Tregs and inhibits Th17 cell differentiation in vitro. Bone marrow-derived macrophages (BMMs) were isolated from WT and ATF3 KO mice and pretreated with Rap or DMSO vehicle controls, and then co-cultured with splenic CD4 ${ }^{+} \mathrm{T}$ cells after LPS stimulation for $6 \mathrm{~h}$. $\mathbf{a}, \mathbf{b}$ Western blot analysis of phosphorylated mTOR, phosphorylated p70S6K, PHD1, HIF-1a, HMGB1, and TLR4 in LPS-stimulated BMMs. $\beta$-actin served as an internal control. c Quantitative RT-PCRassisted detection of TNF- $\alpha$, IL-1 $\beta$, IL-6, and TGF- $\beta$ in LPS-stimulated BMMs. Mean \pm SD ( $n=3-4$ samples/group). ${ }^{*} p<0.05$, ** $p<0.01$. d Quantitative RT-PCR-assisted detection of Foxp3, RORyt, and IL-17A in splenic CD4 ${ }^{+}$T cells after co-culture with Rap or DMSO-pretreated BMMs. Mean \pm SD ( $n=3-4$ samples/group). ${ }^{*} p<0.05,{ }^{* *} p<0.01$. e ELISA analysis of IL-17A levels in co-culture supernatants. Mean \pm SD ( $n=3-4$ samples/group). ${ }^{* *} p<0.01$. $\mathbf{f}$ Western blot analysis of ATF3, phosphorylated mTOR, phosphorylated p70S6K, PHD1, and HIF-1a in LPS-stimulated BMMs followed by Ad-ATF3 or Ad-con-pretreatment. $\beta$-actin served as an internal control. Data are representative of three experiments

in LPS-stimulated BMMs after Ad-ATF3 or Ad-con treatment (Fig. 4f). These results suggested that macrophage ATF3 regulated innate TLR4 and adaptive $\mathrm{T}$ cell differentiation via the mTOR/HIF- $1 \alpha$-mediated signaling pathway.

\section{p70S6K mediates mTOR signaling in ATF3-mediated immune regulation in vitro}

As the phosphorylation of S6K acted downstream of mTOR activation and played an important role in cell metabolism and transcriptional regulation ${ }^{31}$, we next investigated the functional role of macrophage p70S6K in ATF3-mediated immune regulation in cell cultures.
Unlike the non-specific (NS) siRNA-treated controls, siRNA-mediated knockdown of p70S6K (si-p70S6K) in ATF3-deficient BMMs downregulated HMGB1, TLR4, and NF- $\mathrm{B}$, upregulated PHD1, and downregulated HIF- $1 \alpha$ after LPS stimulation (Fig. 5a). Treatment of ATF3-deficient BMMs with si-p70S6K decreased the mRNA levels of TNF- $\alpha$, IL- $1 \beta$, and IL- 6 and increased those of TGF- $\beta$ in response to LPS stimulation compared with the NS siRNA-treated controls (Fig. 5b). Using macrophage $(\mathrm{BMM}) /$ spleen $\mathrm{CD}^{+} \mathrm{T}$ cell co-culture system, p70S6K siRNA treatment in BMMs upregulated Foxp3 and downregulated RORYt and IL-17A expression in spleen $\mathrm{CD}^{+} \mathrm{T}$ cells (Fig. 5c), and this 


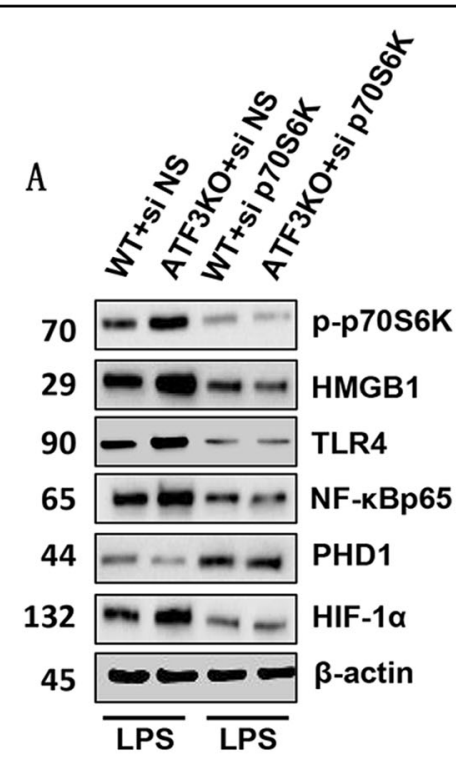

$\mathrm{C}$

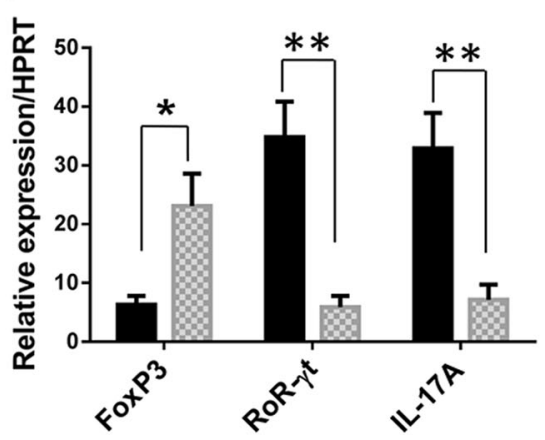

B

LPS+ATF3KO+si NS LPS+ATF3KO+si p70s6K

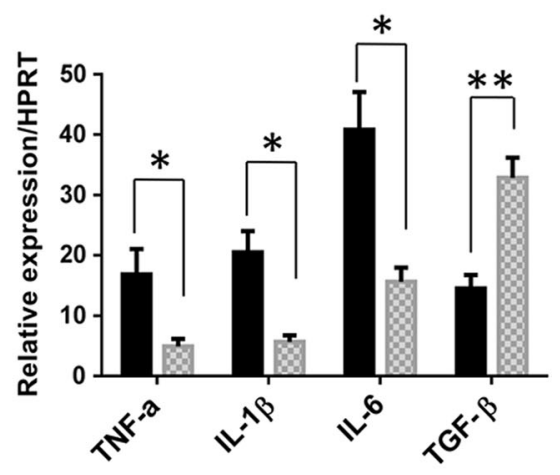

D

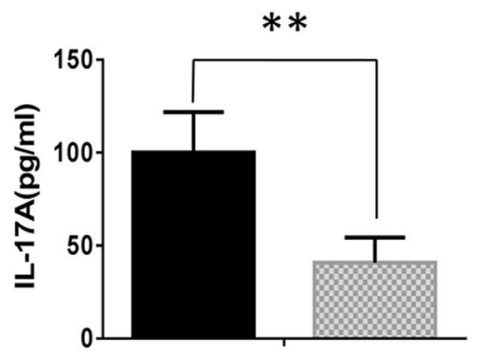

Fig. 5 p70S6K mediates mTOR signaling in ATF3-mediated immune regulation in vitro. Bone marrow-derived macrophages (BMMs) were isolated from WT and ATF3 KO mice and transfected with p70S6K siRNA (si-p70S6K), and then co-cultured with splenic CD4 ${ }^{+} \mathrm{T}_{\text {cells }}$ after LPS stimulation for $6 \mathrm{~h}$. Non-specific (NS) siRNA served as a control. a Western blot analysis of phosphorylated p70S6K, PHD1, HIF-1a, HMGB1, TLR4, and NF-KB in LPS-stimulated BMMs. $\beta$-actin served as an internal control. b Quantitative RT-PCR detection of TNF- $\alpha$, IL-1 $\beta$, IL-6, and TGF- $\beta$ in LPSstimulated BMMs. Mean \pm SD ( $n=3-4$ samples/group). ${ }^{*} p<0.05,{ }^{* *} p<0.01$. c Quantitative RT-PCR detection of Foxp3, RORyt, and IL-17A in splenic $\mathrm{CD}^{+} \mathrm{T}$ cells after co-culture with p70S6K siRNA or NS siRNA-pretreated BMMs. Mean \pm SD $\left(n=3-4\right.$ samples/group). ${ }^{*} p<0.05,{ }^{* *} p<0.01$. d ELISA analysis of IL-17A levels in co-culture supernatants. Mean \pm SD ( $n=3-4$ samples/group). ${ }^{* *} p<0.01$

was accompanied by reduced IL-17A levels after co-culture (Fig. 5d). These data indicated that mTOR contributed to ATF3-mediated immune regulation in a p70S6K-dependent manner.

\section{Disruption of HIF-1a ameliorates ATF3 deficiency- mediated liver damage and inhibits Th17 cell differentiation in vivo}

Having demonstrated the important role of macrophage p70S6K/HIF- $1 \alpha$ signaling in the modulation of innate and adaptive immunity in vitro, we next investigated whether disruption of macrophage HIF-1 $\alpha$ in ATF3 KO mice affected inflammatory responses and $\mathrm{T}$ cell differentiation in mouse liver IRI. An HIF- $1 \alpha$ siRNA with an in vivo mannose-mediated delivery system, which enhances delivery to cells expressing a mannose-specific membrane receptor, was used to transfect to macrophages ${ }^{32}$. First, we assessed the extent of HIF-1a downregulation after HIF$1 \alpha$ siRNA or NS siRNA treatment, which showed that pretreatment with HIF- $1 \alpha$ siRNA markedly decreased the protein levels of HIF-1 $\alpha$ (Fig. 6a). The livers of ATF3 KO mice treated with NS siRNA displayed significant edema, severe sinusoidal congestion/cytoplasmic vacuolization, and extensive necrosis (Fig. 6a, b, score $=2.95 \pm 0.37$ ). However, the livers of ATF3 KO mice treated with mannose-mediated HIF- $1 \alpha$ siRNA showed mild to moderate edema without necrosis (Fig. 6a, b, score $=1.25 \pm$ $0.25, p<0.001)$, and a lower frequency of $\mathrm{TUNEL}^{+}$cells than the NS siRNA-treated controls (Fig. 6a, 83.4 \pm 6.54 vs. $44.6 \pm 4.2, p<0.001)$. These data were consistent with 


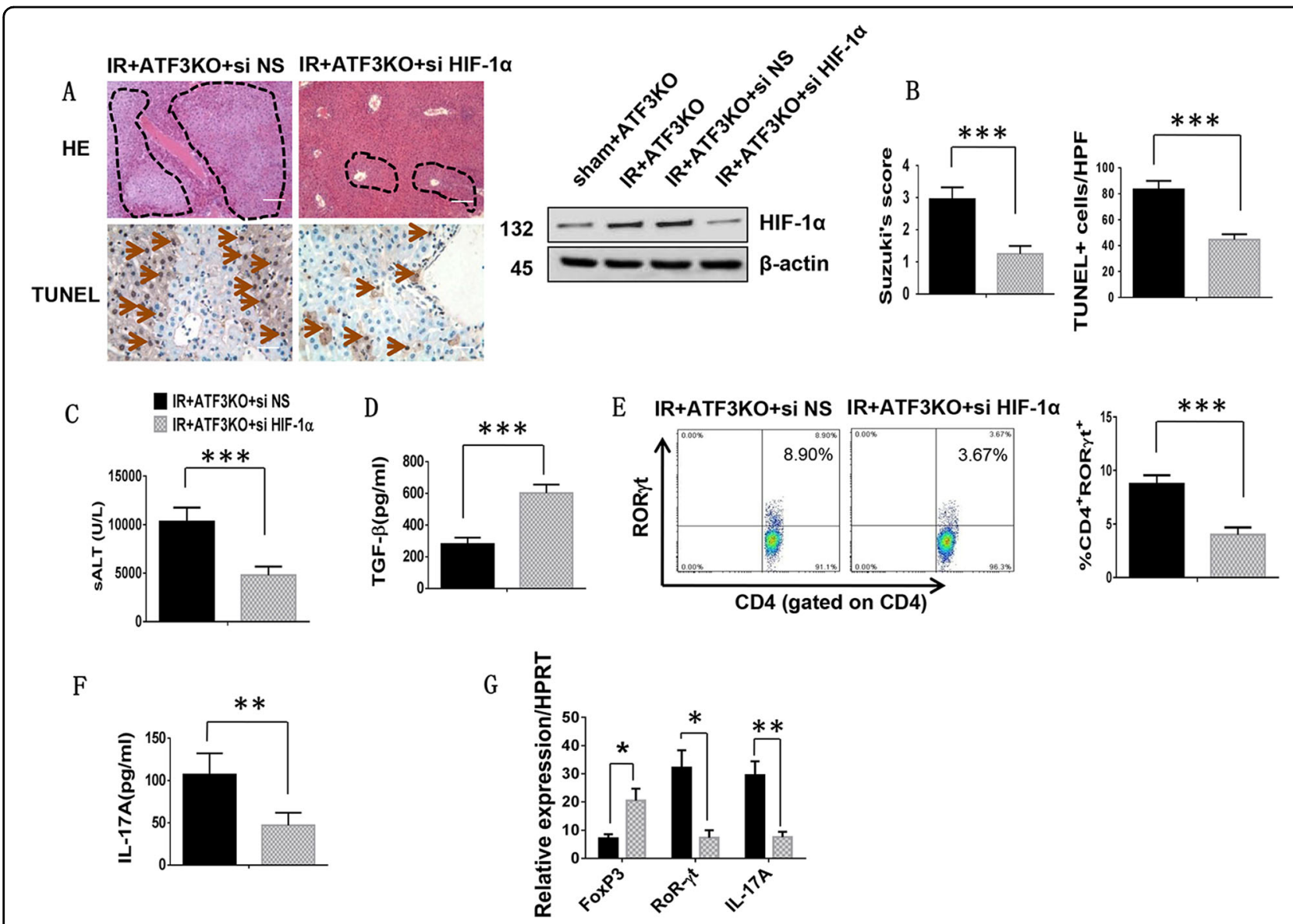

Fig. 6 Disruption of HIF-1 a ameliorates ATF3 deficiency-mediated liver damage and inhibits Th17 cell differentiation in vivo. ATF3 KO mice were injected via the tail vein with a mannose-mediated HIF-1a siRNA or NS siRNA at $4 \mathrm{~h}$ prior to ischemia. a Representative histological staining $(\mathrm{H} \&$ E, original magnification $\times 100)$ and TUNEL staining of ischemic liver tissue (4-6 mice/group). Scale bars $=50 \mu \mathrm{m}$. Western blot analysis of HIF-1a in HIF-1a siRNA or NS siRNA-pretreated livers subjected to IR. $\beta$-actin served as an internal control. b Liver damage, as evaluated by Suzuki's score. ${ }^{* * *} p<0.001$. TUNEL staining, results were scored semi-quantitatively by averaging the number of apoptotic cells (mean \pm SD) per field at $\times 400$ magnification. ${ }^{* * *} p<0.001$. c Hepatocellular function, as assessed by serum ALT levels (IU/L). Results are expressed as the mean \pm SD ( $n=$ 4-6 samples/group). ${ }^{* *} p<0.001$. d ELISA analysis of serum TGF- $\beta$ levels. Mean \pm SD ( $n=3-4$ samples/group). ${ }^{* * *} p<0.001$. e RORyt expression in spleen $T$ cells was evaluated by flow cytometry. Representative of three separate experiments. ${ }^{* * *} p<0.001$. $\mathbf{f}$ ELISA analysis of serum IL-17A levels. Mean \pm SD ( $n=3-4$ samples/group). ${ }^{* *} p<0.01$. g Foxp3, RORyt, and IL-17A in mouse livers. Mean \pm SD $\left(n=3-4\right.$ samples/group). ${ }^{*} p<0.05$, ** $p<0.01$

the results of hepatocellular function analysis, which showed that mannose-mediated HIF-1 $\alpha$ siRNA treatment in ATF3 KO mice decreased sALT levels compared with those in the NS siRNA-treated controls (Fig. 6c, 10,304 \pm 1449 vs. $4798 \pm 883, p<0.001$ ). Moreover, HIF- $1 \alpha$ siRNA treatment in ATF3 KO livers increased serum TGF- $\beta$ release (Fig. 6 d, $281.2 \pm 39.55$ vs. $602.6 \pm 53.04, p<0.001$ ), and this was accompanied by a reduction in the percentage of splenic CD4 ${ }^{+}$RoRyt $^{+}$TH17 cells (Fig. 6e, 8.74 \pm 0.82 vs. $4.01 \pm 0.67, p<0.001)$ and serum IL-17A levels (Fig. 6f, $107 \pm 25.2$ vs. $47 \pm 14.9, p=0.009$ ) compared with the NS siRNA-treated controls. RORyt and IL-17A mRNA levels were reduced, whereas Foxp3 levels were increased in HIF- $1 \alpha$ siRNA-treated groups but not the NS siRNA-treated controls (Fig. 6g). These results suggested that macrophage HIF-1 $\alpha$ signaling was essential for modulating Th17 cell differentiation and inflammatory responses in ATF3-mediated immune regulation (Fig. 7).

\section{Discussion}

The present study is the first to demonstrate that ATF3mediated mTOR/p70S6K/HIF-1 $\alpha$ signaling is crucial for orchestrating inflammatory responses in IR-induced liver injury. The data can be summarized as follows: (i) ATF3 deficiency exacerbated IR-induced liver damage, increased macrophage/neutrophil trafficking, promoted mTOR and its downstream p70S6K, and activated TLR4/ NF- $\mathrm{kB}$; and (ii) ATF3-mediated mTOR/p70S6K induced HIF- $1 \alpha$ signaling, which was essential for $\mathrm{T}$ cell differentiation in liver IRI. These results highlighted the role of 


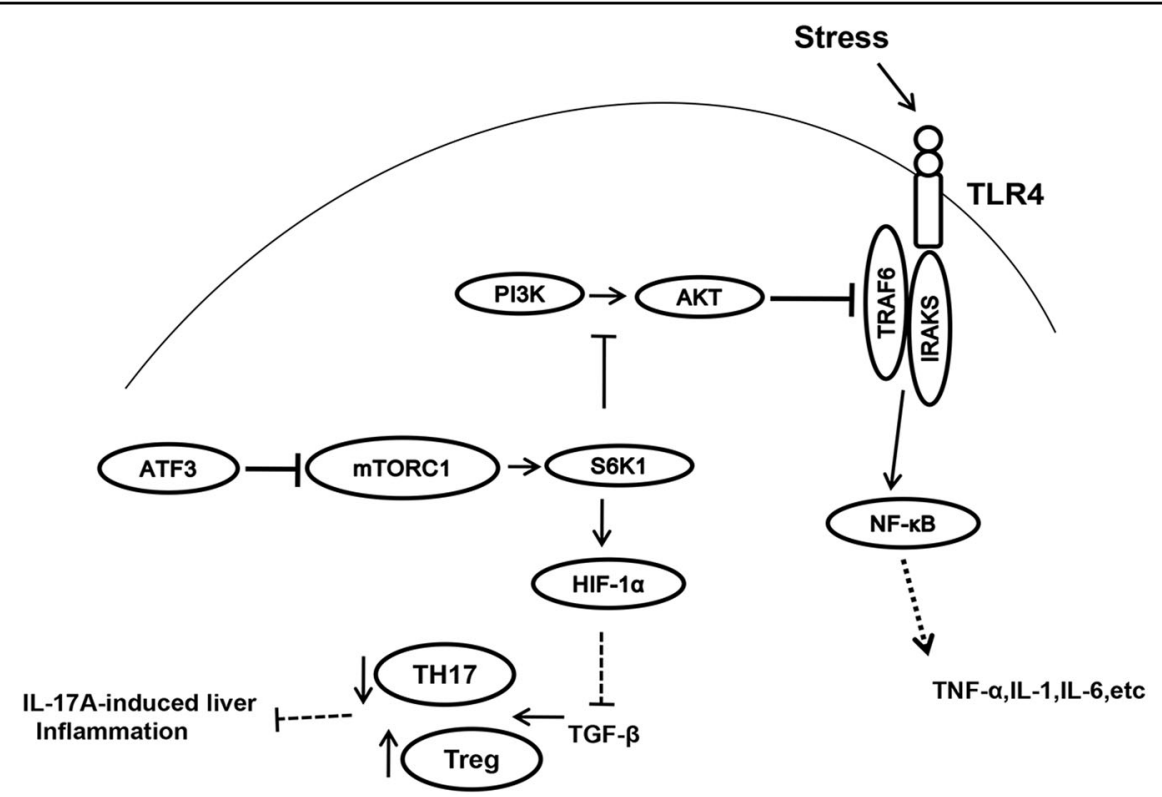

Fig. 7 Schematic illustration of the molecular mechanisms underlying the role of mTOR/p70S6K/HIF-1a signaling pathway in the regulation of TLR4 inflammatory signaling and TH17 cells in liver IRI

ATF3-mediated mTOR/p70S6K/HIF-1 $\alpha$ signaling in the regulation of innate and adaptive immunity during liver inflammatory injury.

ATF3, an oxidative stress-responsive transcription factor, is associated with a variety of immune and inflammatory diseases ${ }^{13,33}$. Although previous studies showed that ATF3 modulates inflammatory responses by inhibiting TLR4-mediated inflammatory cytokines ${ }^{34,35}$, little is known about the exact mechanism by which ATF3 regulates innate TLR4 and adaptive $\mathrm{T}$ cell differentiation in IR-induced liver injury. In addition, ATF3 has multiple roles in neuroprotection and neuroregeneration ${ }^{36,37}$. In the current study, ATF3 deletion increased the inflammatory response, as evidenced by the exacerbation of IR-induced liver damage and increased hepatocellular apoptosis and intrahepatic macrophage/neutrophil accumulation. Moreover, ATF3 deficiency activated mTOR, p70S6K, TLR4, and HIF-1 $\alpha$, and downregulated PHD1. Disruption of ATF3 downregulated Foxp3 and upregulated RORyt-mediated IL-17A expression in IR-induced liver inflammation. These results underscored the importance of ATF3 as a negative transcriptional regulator of innate TLR4 and T cell differentiation during liver inflammatory injury.

The molecular mechanisms underlying the role of ATF3 in regulating innate TLR4 and adaptive $\mathrm{T}$ cell differentiation may involve in multiple cellular and molecular signaling pathways. mTOR, serine/threonine kinase, function as a core component of two distinct protein complexes, mTORC1 and mTORC2, which regulate different cellular functions ${ }^{38}$, including cell growth, lipogenesis, protein synthesis, and transcription ${ }^{39,40}$. Constitutive mTORC1 activation promotes M1 macrophage polarization and increases inflammatory responses after LPS stimulation ${ }^{19,20}$. Activation of mTORC1 upregulates TLR4 signaling and pro-inflammatory cytokines in acute lung injury ${ }^{41}$. Moreover, mTOR is essential for $\mathrm{T}$ cell proliferation and differentiation ${ }^{42-46}$. The close association of ATF3 with mTOR was reported previously ${ }^{47}$. Consistent with these results, we found that ATF3 knockout promoted mTOR activity, whereas inhibition of mTOR ameliorated IR-induced liver injury and reduced HMGB1 and TLR4 activation, suggesting that mTOR signaling mediated the role of ATF3 in the regulation of innate TLR4 during liver IRI.

Further evidence of mTOR signaling-mediated modulation of innate TLR4 was obtained from our in vitro study. The results showed that ATF3 deficiency promoted mTOR and its downstream target gene p70S6K phosphorylation in LPS-stimulated BMMs. However, inhibition of mTOR in ATF3-deficient BMMs reduced p70S6K activity and downregulated HMGB1 and TLR4, and this was accompanied by the downregulation of proinflammatory cytokines. Indeed, p70S6K functions as part of the mTOR signaling pathway. Phosphorylation of p70S6K is dependent on mTOR, specifically on mTORC1. The mTORC1-S6K axis controls a variety of cellular processes and contributes to cell development and disease ${ }^{31}$. Disruption of TSC-mediated mTOR/S6K signaling is associated with several human genetic disorders $^{48}$. Consistent with these findings, we found that disruption of p70S6K in ATF3-deficient BMMs inhibited 
TLR4-driven inflammatory responses after LPS stimulation. Notably, ATF3 deletion in LPS-stimulated BMMs reduced PHD1 activity and increased HIF- $1 \alpha$ induction, whereas p70S6K knockdown upregulated PHD1 and downregulated HIF- $1 \alpha$, thus decreasing pro-inflammatory mediators in ATF3-deficient cells. These results suggested that ATF3-mediated mTOR/p70S6K signaling positively regulated HIF- $1 \alpha$ activity during the inflammatory response.

One striking finding was that ATF3 deficiency depressed Foxp $3^{+}$Tregs, whereas it increased $\mathrm{ROR}^{+}$Th17 cells in IR-induced liver inflammation. Although previous studies showed that mTOR is a major regulator of T cell differentiation and expansion ${ }^{49}$, how macrophage ATF3 affects $\mathrm{T}$ cell differentiation remains unknown. In the present macrophage/CD4 ${ }^{+} \mathrm{T}$ cell co-culture system, increased HIF- $1 \alpha$ induction in ATF3-deficient BMMs upregulated ROR $\gamma \mathrm{t}$ and IL-17A and downregulated Foxp3 expression in splenic $\mathrm{CD} 4^{+} \mathrm{T}$ cells, and this was accompanied by increased IL-17A production. However, inhibition of mTOR by rapamycin in ATF3-deficient BMMs upregulated PHD1 and downregulated HIF- $1 \alpha$, resulting in increased Foxp3 and diminished RORyt and IL-17A levels. Indeed, HIF- $1 \alpha$ can regulate innate and adaptive immune cell functions. Ablation of myeloid-specific HIF-1 $\alpha$ suppresses inflammatory responses by inhibiting macrophage infiltration and activation ${ }^{27}$. The contribution of HIF- $1 \alpha$ to the inflammatory response is dependent on NF- $\mathrm{kB}$ activity ${ }^{50}$. Moreover, HIF-1 $\alpha$ promotes Th17 cell development by activating ROR $\gamma \mathrm{t}$ transcription, whereas deletion of HIF- $1 \alpha$ in T cells promotes Foxp $3^{+}$ Tregs and decreased RORyt ${ }^{+}$Th17 cells ${ }^{28}$. Consistent with these results, we found that disruption of HIF- $1 \alpha$ in ATF3 KO mice alleviated IR-induced liver damage and improved hepatic function, and this occurred in parallel with reduced RORyt-mediated Th17A levels and increased Foxp3 expression. Thus, our findings revealed an essential role for HIF- $1 \alpha$ in the control of T cell differentiation in ATF3-mediated immune regulation during liver inflammatory injury.

The effect of macrophage ATF3-mediated mTOR signaling on the ability of HIF- $1 \alpha$ to regulate T cell differentiation remains unclear. We showed that ATF3 deficiency increased HIF- $1 \alpha$ induction while reducing PHD1 activity. However, inhibition of mTOR in ATF3 KO mice reduced phosphorylated p70S6K and HIF- $1 \alpha$ and increased PHD1 in ischemic livers. This suggested a possible mechanistic link between mTOR and HIF- $1 \alpha$ in the regulation of $\mathrm{T}$ cell differentiation? Indeed, HIF- $1 \alpha$ stability is primarily modulated by PHD1 in an oxygendependent-manner. PHD1 acts as an oxygen-sensing enzyme and promotes HIF- $1 \alpha$ hydroxylation and proteasomal degradation in normoxia, whereas inactivated PHD1 during hypoxia leads to the stabilization of HIF- $1 \alpha$ and its translocation into the nucleus to activate the transcription of target genes ${ }^{51}$. Thus, we speculate that ATF3-mediated mTOR signaling may play an important role in the regulation of the HIF-1 $\alpha$-PHD1 oxygensensing pathway. As p70S6K activation is modulated by mTOR, p70S6K may be essential for the regulation of HIF- $1 \alpha$ induction in the mechanism of adaptive $\mathrm{T}$ cell development. This was supported by our further experiments. We used a co-culture system to show that knockdown of p70S6K in ATF3-deficient BMMs increased PHD1 and reduced HIF- $1 \alpha$ activity, and this was accompanied by increased Foxp3 and decreased RORyt-mediated IL-17A levels in splenic CD4 ${ }^{+} \mathrm{T}$ cells. Taken together, these data indicated that ATF3-mediated mTOR/p70S6K/HIF- $1 \alpha$ signaling was crucial for $\mathrm{T}$ cell differentiation in IR-triggered liver inflammation.

In conclusion, we demonstrated that ATF3 deficiency exacerbated IR-induced liver inflammation by upregulating mTOR and its downstream target gene p70S6K, which in turn activated innate TLR4 and increased HIF- $1 \alpha$ while reducing PHD1 activity, leading to depressed Foxp3 ${ }^{+}$ Treg and promoting ROR $\mathrm{t}^{+}$Th17 cell differentiation. The present study has increased our knowledge of the molecular mechanisms underlying the role of ATF3mediated mTOR/p70S6K//HIF-1 $\alpha$ signaling in the modulation of innate TLR4 and adaptive $\mathrm{T}$ cell differentiation, thus providing potential therapeutic targets in liver IRI followed by liver transplantation.

\section{Materials and methods \\ Animals}

WT C57BL/6 mice were purchased from the Laboratory Animal Resources of Nanjing Medical University (NMU). ATF3 knockout (KO) mice in the C57BL/6 background were previously described ${ }^{34}$. Male, 8-week-old WT and ATF3 KO mice were used in all experiments. This study was performed in strict accordance with the recommendations in the Guide for the Care and Use of Laboratory Animals published by the National Institutes of Health. The animal protocol was approved by the Institutional Animal Care \& Use Committee (IACUC) of Nanjing Medical University (Protocol Number NMU08-092).

\section{Mouse liver IRI model}

A mouse model of warm hepatic ischemia followed by reperfusion was used, as described ${ }^{30}$. Mice were injected with heparin $(100 \mathrm{U} / \mathrm{kg})$ and an atraumatic clip was used to interrupt the arterial/portal venous blood supply to the cephalad liver lobes. After $90 \mathrm{~min}$ the clip was removed, and mice were killed at $6 \mathrm{~h}$ of reperfusion. Mice were injected with mTOR inhibitor Rapamycin $(5 \mathrm{mg} / \mathrm{kg}$, i.p. Calbiochem, Burlington, MA) or DMSO vehicle at $60 \mathrm{~min}$ prior to ischemia. In some experiments, animals were injected via tail vein with HIF- $1 \alpha$ siRNAs or non-specific 
(control) siRNA, (2 mg/kg) (Santa Cruz Biotechnology, Shanghai, China) mixed with mannose-conjugated polymers (Polyplus transfection ${ }^{\mathrm{Tm}}$, Illkirch, France) at a ratio according to the manufacturer's instructions $4 \mathrm{~h}$ prior to ischemia as described ${ }^{32}$.

\section{Hepatocellular function assay}

Serum alanine aminotransferase (sALT) levels, an indicator of hepatocellular injury, were measured by an automated chemical analyzer (Olympus Automated Chemistry Analyzer AU5400, Tokyo, Japan).

\section{Histology}

Liver sections were stained with hematoxylin and eosin (H\&E). The severity of IRI was graded using Suzuki's criteria on a scale from 0 to $4^{52}$. In this classification, no necrosis, congestion, or centrilobular ballooning is given a score of 0 , while severe congestion and ballooning degeneration and $>60 \%$ lobular necrosis is given a value of 4 .

\section{Immunohistochemistry staining}

Liver macrophages and neutrophils were detected using primary rat anti-mouse $\mathrm{CD}_{11 \mathrm{~b}^{+}} \mathrm{mAb}$ (Mac-1, M1/70; BD Biosciences, San Jose, CA) or $\mathrm{Ly}^{+} \mathrm{G}^{+}$mAb (BD Biosciences, San Diego, CA). After incubation with secondary biotinylated goat anti-rat IgG (Vector, Burlingame, CA), followed by treatment with immunoperoxidase (ABC Kit, Vector), positive cells were counted blindly in ten HPF/ section $(\times 400)$. For immunofluorescence, frozen liver sections were labeled with primary antibodies ATF3 and CD11b (Santa Cruz Biotechnology, CA), and then incubated with secondary Cy3-conjugated AffiniPure donkey anti-goat IgG antibody (Jackson Immunoresearch, PA). The samples were pre-mounted with VECTASHIELD medium with DAPI.

\section{Myeloperoxidase activity assay}

The presence of myeloperoxidase (MPO) was used as an index of hepatic neutrophil accumulation ${ }^{2}$. The change in absorbance was measured spectrophotometrically at $655 \mathrm{~nm}$. One unit of MPO activity was defined as the quantity of enzyme degrading $1 \mu \mathrm{mol}$ peroxide/min at $25^{\circ} \mathrm{C} / \mathrm{g}$ of tissue.

\section{TUNEL staining}

Liver sections $(4 \mathrm{~mm})$ were stained via terminal deoxynucleotidyl transferase dUTP nick end labeling (TUNEL) using the in situ cell death detection kit (Roche-Boehringer Mannheim, Germany) according to the manufacturer's instructions as previously described ${ }^{2}$.

\section{Caspase- 3 activity assay}

Caspase-3 activity was determined by an assay kit (Calbiochem, La Jolla, CA), as previously described ${ }^{2}$. Liver tissues were collected and resuspended in lysis buffer containing $50 \mathrm{mmol} / \mathrm{L}$ HEPES, pH 7.4, 0.1\% CHAPS, 1 $\mathrm{mmol} / \mathrm{L}$ DTT, $0.1 \mathrm{mmol} / \mathrm{L}$ EDTA, and $0.1 \%$ Triton X-100. Following incubation for $30 \mathrm{~min}$ on ice, cell lysate was centrifuged at $16,000 \times g$ for $10 \mathrm{~min}$ at $4{ }^{\circ} \mathrm{C}$, and the protein concentration in the supernatants was measured using the Bradford dye method. The supernatants were incubated with $200 \mu \mathrm{M}$ of enzyme-specific colorimetric caspase- 3 substrate at $37^{\circ} \mathrm{C}$ for $2 \mathrm{~h}$. Caspase- 3 activity was assessed by measuring the absorbance at a wavelength of $405 \mathrm{~nm}$ with a plate reader. To determine cellular activity, the inhibitor-treated protein extracts and the purified caspase-3 (as a standard) were used.

\section{ELISA}

IL-17A and TGF- $\beta$ levels were measured by ELISA according to the manufacturer's standard protocols (eBioscience, San Diego, CA). Absorbance was read on a Multiscan FC plate reader and analyzed with SkanIt for Multiscan FC software (Thermo Scientific, Schwerte, Germany).

\section{Quantitative RT-PCR analysis}

Quantitative real-time PCR was performed using the DNA Engine with Chromo 4 Detector (MJ Research, Waltham, MA). In a final reaction volume of $25 \mu \mathrm{l}$, the following were added: $1 \times$ SuperMix (Platinum SYBR Green qPCR Kit; Invitrogen, San Diego, CA) cDNA and $10 \mu \mathrm{M}$ of each primer. Amplification conditions were: $50{ }^{\circ} \mathrm{C}(2 \mathrm{~min}), 95^{\circ} \mathrm{C}(5 \mathrm{~min})$, followed by 40 cycles of $95^{\circ} \mathrm{C}$ $(15 \mathrm{~s})$ and $60^{\circ} \mathrm{C}(30 \mathrm{~s})$. Primer sequences used for the amplification were shown in Supplementary Table 1.

\section{Western blot analysis}

Protein was extracted from liver tissue or cell cultures, as described ${ }^{53}$. Monoclonal rabbit anti-mouse ATF3 (Santa Cruz Biotechnology, Shanghai, China), phosmTOR, mTOR, phos-p70S6K, p70S6K, HMGB1, TLR4, NF-kB, HIF-1 $\alpha$, PHD1, Bcl-2, Bcl-xl, and $\beta$-actin Abs (Cell Signaling Technology, San Diego, CA) were used. The relative quantities of proteins were determined by densitometer, and expressed in absorbance units (AU).

\section{BMM isolation and in vitro transfection}

Murine bone marrow-derived macrophages (BMMs) were generated as previously described ${ }^{54}$. In brief, bone marrow cells were removed from the femurs and tibias of WT and ATF3 KO mice and cultured in DMEM supplemented with 10\% FCS and 20\% L929-conditioned medium. Cells $\left(1 \times 10^{6} /\right.$ well $)$ were cultured for 7 days and then transfected with $100 \mathrm{nM}$ of p70S6K siRNA (Santa Cruz Biotechnology) using lipofectamine 2000 reagent (Thermo Fish Scientific, Carlsbad, CA). The nonspecific (NS) siRNA as controls. After 24-48 h, cells were 
supplemented with $100 \mathrm{ng} / \mathrm{ml}$ of LPS for additional $6 \mathrm{~h}$. In some experiments, BMMs were pretreated with $20 \mathrm{nM}$ of rapamycin (Calbiochem) or DMSO vehicle at $60 \mathrm{~min}$ prior to LPS stimulation.

\section{Spleen $\mathrm{CD}^{+}{ }^{+}$cell isolation}

Spleen $\mathrm{T}$ cells were purified using the EasySep ${ }^{\mathrm{Tm}}$ mouse $\mathrm{T}$ cell isolation kit (STEMCELL Technologies, Vancouver, BC, Canada) according to the manufacturer's instructions. $\mathrm{T}$ cells were then stimulated with anti-CD3 $(1 \mu \mathrm{g} / \mathrm{ml})$ and anti-CD28 $(2 \mu \mathrm{g} / \mathrm{ml})$ (eBioscience). $\mathrm{CD}^{+} \mathrm{T}$ cells were isolated from these cells using anti-CD4 microbeads (Miltenyi Biotec, Bergisch Gladbach, Germany) according to the manufacturer's instructions.

\section{Macrophage $/ \mathrm{CD}^{+} \mathrm{T}$ cell co-culture}

Rapamycin or p70S6K siRNA pretreated ATF3 KO macrophages were counted to $0.5 \times 10^{6}$ cells $/ \mathrm{ml}$ and cultured on $60 \mathrm{~mm}$ plates. After the cells stimulated with LPS $(100 \mathrm{ng} / \mathrm{ml})$ for $6 \mathrm{~h}$, spleen $\mathrm{CD} 4^{+} \mathrm{T}$ cells were then added into cultures at a macrophage/ $\mathrm{T}$ cell ratio of 1:5. The cocultured cells were incubated for $24 \mathrm{~h}$, and then macrophages and spleen $\mathrm{CD} 4^{+} \mathrm{T}$ cells were harvested for the real-time PCR and western blot assay.

\section{Flow cytometry analysis}

Spleen T cells isolated from WT, ATF3 KO, and HIF- $1 \alpha$ siRNA or NS siRNA-treated ATF3 KO mice were stained with anti-mouse CD4-PE-Cyanine5, CD25-PE, RoRyt-PE, and Foxp3-FITC mAbs (eBioscience) according to the manufacturer's instructions. PE-labeled rat anti-mouse IgG2a isotypes were used as negative controls. Measurements were performed using a FACS Calibur flow cytometer (BD Biosciences). Data analysis was performed using Cell Quest software. Liver NPCs were isolated from sham or IR livers, as described above ${ }^{55}$. A total of $1 \times 10^{6}$ cells were incubated with purified rat anti-mouse CD16/ 32 for $10 \mathrm{~min}$ and stained with rat anti-mouse F4/80PeCy5/PE, CD11b-FITC, and isotype-matched negative control Abs (eBioscience, San Diego, CA) were added to the cell suspension. After 20 min of incubation in the dark, the cells were washed with PBS and subjected to flow cytometric analysis with FACS Calibur (BD Biosciences). For intracellular staining of CD206 and inducible NO synthase, cells were fixed in $4 \%$ formaldehyde for 20 min after the staining of F4/80 and CD11b, and washed twice with $1 \times$ permeabilization buffer (eBioscience). After incubation with CD206-APC (BioLegend, San Diego, $\mathrm{CA}$ ) and inducible NO synthase-PE (eBioscience) in $1 \times$ permeabilization buffer for $20 \mathrm{~min}$ in the dark, the cells were washed with PBS and subjected to flow cytometric analysis.

\section{Adenovirus gene transfer}

Adenoviral vector encoding the mouse ATF3 gene (Ad-ATF3) and negative control (Ad-con) was constructed, packaged, purified, and titrated at Genechem Co. Ltd. For adenovirus-mediated gene transfer, Ad-ATF3 or Ad-con was transfected into macrophages from WT mice at a final concentration of $0.5 \times 10^{6}$ cells $/ \mathrm{ml}$ for $48 \mathrm{~h}$. After $48 \mathrm{~h}$, the overexpression efficiency of Ad-ATF3 was evaluated by western blot.

\section{Statistical analysis}

Data are expressed as mean \pm SD and analyzed by Student's $t$ tests. Per comparison, two-sided $p$ values less than 0.05 were considered statistically significant. Multiple group comparisons were performed using one-way ANOVA with a post hoc test. All statistical analysis was performed using SPSS-3 software.

\section{Acknowledgements}

This work was supported by grants from the National Natural Science Foundation of China 81100270, 1310108001, 81210108017, National Science Foundation of Jiangsu Province BK20131024, BE2016766, Major project of Nanjing Medical University 2014NJMUZD081, 863 Young Scientists Special Fund grant SS2015AA0209322 and the Foundation of Jiangsu Collaborative Innovation Center of Biomedical Functional Materials.

\section{Author details}

${ }^{1}$ Liver Transplantation Center, First Affiliated Hospital, Nanjing Medical University, Nanjing, China. ${ }^{2}$ Children's Hospital of Nanjing Medical University, Nanjing, China. ${ }^{3}$ Department of Physiology, School of Basic Medical Sciences, Wuhan University, Wuhan, China. ${ }^{4}$ The Dumont-UCLA Transplant Center, Division of Liver and Pancreas Transplantation, Department of Surgery, David Geffen School of Medicine at University of California-Los Angeles, Los Angeles, CA, USA

\section{Authors' contributions}

Q.Z. and H.W. performed in vitro and in vivo experiments and analyzed the data. Q.Z. wrote the first draft of manuscript; X.N. performed in vivo experiments; L.J. and C.L. performed in vitro experiments; X.W., F.Z., and B.J. participated in data analysis and critical discussion; B.K. and L.L. contributed to the study concept, research design, and finalized the manuscript. All authors (Q.Z., H.W., X.N., L.J., C.L., X.W., F.Z., B.J., B.K., and L.L.) were involved in data interpretation, technical support, and writing the paper, and had final approval of the submitted and published versions.

Conflict of interest

The authors declare that they have no conflict of interest.

\section{Publisher's note}

Springer Nature remains neutral with regard to jurisdictional claims in published maps and institutional affiliations.

Supplementary Information accompanies this paper at (https://doi.org/ 10.1038/s41419-018-0894-1).

Received: 9 March 2018 Revised: 10 July 2018 Accepted: 11 July 2018 Published online: 05 September 2018

\footnotetext{
References

1. Lu, L. et al. Innate immune regulations and liver ischemia-reperfusion injury. Transplantation 100, 2601-2610 (2016).
} 
2. Ke, B. et al. HO-1-STAT3 axis in mouse liver ischemia/reperfusion injury: regulation of TLR4 innate responses through PI3K/PTEN signaling. J. Hepatol. 56, 359-366 (2012).

3. Jaeschke, H., Smith, C. V. \& Mitchell, J. R. Reactive oxygen species during ischemia-reflow injury in isolated perfused rat liver. J. Clin. Invest. 81, 1240-1246 (1988).

4. Colletti, L. M. et al. Role of tumor necrosis factor-alpha in the pathophysiologic alterations after hepatic ischemia/reperfusion injury in the rat. J. Clin. Invest. $\mathbf{8 5}$ 1936-1943 (1990).

5. Zwacka, R. M. et al. CD4(+) T-lymphocytes mediate ischemia/reperfusioninduced inflammatory responses in mouse liver. J. Clin. Invest. 100, 279-289 (1997).

6. Harrington, L. E. et al. Interleukin 17-producing CD4+effector T cells develop via a lineage distinct from the T helper type 1 and 2 lineages. Nat. Immunol. $\mathbf{6}$, 1123-1132 (2005).

7. Dong, C. TH17 cells in development: an updated view of their molecular identity and genetic programming. Nat. Rev. Immunol. 8, 337-348 (2008).

8. Eggenhofer, E. et al. Unconventional RORgammat+T cells drive hepatic ischemia reperfusion injury. J. Immunol. 191, 480-487 (2013).

9. Zhu, Q. et al. Phosphatase and tensin homolog-beta-catenin signaling modulates regulatory $T$ cells and inflammatory responses in mouse liver ischemia/reperfusion injury. Liver Transpl. 23, 813-825 (2017).

10. Song, D. Y. et al. Role of activating transcription factor 3 in ischemic penumbra region following transient middle cerebral artery occlusion and reperfusion injury. Neurosci. Res. 70, 428-434 (2011).

11. Allen-Jennings, A. E., Hartman, M. G., Kociba, G. J. \& Hai, T. The roles of ATF3 in glucose homeostasis. A transgenic mouse model with liver dysfunction and defects in endocrine pancreas. J. Biol. Chem. 276, 29507-29514 (2001).

12. Hai, T., Wolfgang, C. D., Marsee, D. K., Allen, A. E. \& Sivaprasad, U. ATF3 and stress responses. Gene Expr. 7, 321-335 (1999).

13. Yoshida, T. et al. ATF3 protects against renal ischemia-reperfusion injury. J. Am. Soc. Nephrol. 19, 217-224 (2008).

14. Li, H. F., Cheng, C. F., Liao, W. J., Lin, H. \& Yang, R. B. ATF3-mediated epigenetic regulation protects against acute kidney injury. J. Am. Soc. Nephrol. 21, 1003-1013 (2010).

15. Wang, L. et al. Increased inflammation and brain injury after transient focal cerebral ischemia in activating transcription factor 3 knockout mice. Neuroscience 220, 100-108 (2012).

16. Gilchrist, M. et al. Systems biology approaches identify ATF3 as a negative regulator of Toll-like receptor 4. Nature 441, 173-178 (2006).

17. Laplante, M. \& Sabatini, D. M. mTOR signaling at a glance. J. Cell Sci. 122 3589-3594 (2009)

18. Bhaskar, P. T. \& Hay, N. The two TORCs and Akt. Dev. Cell 12, 487-502 (2007)

19. Zhu, L. et al. TSC1 controls macrophage polarization to prevent inflammatory disease. Nat. Commun. 5, 4696 (2014).

20. Byles, V. et al. The TSC-mTOR pathway regulates macrophage polarization. Nat Commun. 4, 2834 (2013).

21. Bao, W. et al. mTORC1 regulates flagellin-induced inflammatory response in macrophages. PLOS ONE 10, e0125910 (2015).

22. Zeng, $H$. et al. mTORC1 and mTORC2 kinase signaling and glucose metabolism drive follicular helper $\mathrm{T}$ cell differentiation. Immunity 45, 540-554 (2016).

23. Chi, H. Regulation and function of mTOR signalling in T cell fate decisions. Nat Rev. Immunol. 12, 325-338 (2012).

24. Kim, J. S. et al. Natural and inducible $\mathrm{TH} 17$ cells are regulated differently by Akt and mTOR pathways. Nat. Immunol. 14, 611-618 (2013).

25. Semenza, G. L. Hypoxia-inducible factors in physiology and medicine. Cell $\mathbf{1 4 8}$ 399-408 (2012)

26. Epstein, A. C. et al. C. elegans EGL-9 and mammalian homologs define a family of dioxygenases that regulate HIF by prolyl hydroxylation. Cell 107, 43-54 (2001).

27. Cramer, T. et al. HIF-1alpha is essential for myeloid cell-mediated inflammation. Cell 112, 645-657 (2003)

28. Dang, E. V. et al. Control of $\mathrm{T}(\mathrm{H}) 17 / \mathrm{T}(\mathrm{reg})$ balance by hypoxia-inducible factor 1 . Cell 146, 772-784 (2011).

29. Land, S. C. \& Tee, A. R. Hypoxia-inducible factor 1alpha is regulated by the mammalian target of rapamycin (mTOR) via an mTOR signaling motif. J. Biol. Chem. 282, 20534-20543 (2007).
30. Shen, X. D. et al. CD154-CD40 T-cell costimulation pathway is required in the mechanism of hepatic ischemia/reperfusion injury, and its blockade facilitates and depends on heme oxygenase-1 mediated cytoprotection. Transplantation 74, 315-319 (2002)

31. Magnuson, B., Ekim, B. \& Fingar, D. C. Regulation and function of ribosomal protein S6 kinase (S6K) within mTOR signalling networks. Biochem. J. 441, 1-21 (2012).

32. Lu, L. et al. Myeloid notch1 deficiency activates RhoAROCK pathway and aggravates hepatocellular damage in mouse ischemic livers. Hepatology 67, 1041-1055 (2018).

33. Hartman, M. G. et al. Role for activating transcription factor 3 in stress-induced beta-cell apoptosis. Mol. Cell. Biol. 24, 5721-5732 (2004).

34. Rao, J. et al. ATF3-mediated NRF2/HO-1 signaling regulates TLR4 innate immune responses in mouse liver ischemia/reperfusion injury. Am. J. Transplant. 15, 76-87 (2015).

35. Kwon, J. W. et al. Activating transcription factor 3 represses inflammatory responses by binding to the p65 subunit of NF-kappaB. Sci. Rep. 5, 14470 (2015).

36. Gey, M. et al. Atf3 mutant mice show reduced axon regeneration and impaired regeneration-associated gene induction after peripheral nerve injury. Open Biol. 6 (2016). pii: 160091. https://doi.org/10.1098/rsob.160091.

37. Chandrasekar, A. et al. Neuroprotective effect of acute ethanol intoxication in $\mathrm{TBI}$ is associated to the hierarchical modulation of early transcriptional responses. Exp. Neurol. 302, 34-45 (2018).

38. Laplante, M. \& Sabatini, D. M. mTOR signaling in growth control and disease. Cell 149, 274-293 (2012)

39. Howell, J. J. \& Manning, B. D. mTOR couples cellular nutrient sensing to organismal metabolic homeostasis. Trends Endocrinol. Metab. 22, 94-102 (2011).

40. Duvel, K. et al. Activation of a metabolic gene regulatory network downstream of mTOR complex 1. Mol. Cell 39, 171-183 (2010).

41. Lorne, E. et al. Participation of mammalian target of rapamycin complex 1 in Toll-like receptor 2- and 4-induced neutrophil activation and acute lung injury. Am. J. Respir. Cell Mol. Biol. 41, 237-245 (2009).

42. $\mathrm{Li}, \mathrm{Q}$ et al. A central role for mTOR kinase in homeostatic proliferation induced CD8+T cell memory and tumor immunity. Immunity 34, 541-553 (2011).

43. Jones, R. G. \& Thompson, C. B. Revving the engine: signal transduction fuels T cell activation. Immunity 27, 173-178 (2007).

44. Fox, C. J., Hammerman, P. S. \& Thompson, C. B. Fuel feeds function: energy metabolism and the T-cell response. Nat. Rev. Immunol. 5, 844-852 (2005).

45. Powell, J. D. \& Delgoffe, G. M. The mammalian target of rapamycin: linking T cell differentiation, function, and metabolism. Immunity 33, 301-311 (2010).

46. Zheng, $Y$. et al. A role for mammalian target of rapamycin in regulating $T$ cell activation versus anergy. J. Immunol. 178, 2163-2170 (2007).

47. Nie, D. et al. The stress-induced Atf3-gelsolin cascade underlies dendritic spine deficits in neuronal models of tuberous sclerosis complex. J. Neurosci. 35 10762-10772 (2015)

48. Inoki, K., Corradetti, M. N. \& Guan, K. L. Dysregulation of the TSC-mTOR pathway in human disease. Nat. Genet. 37, 19-24 (2005).

49. Pollizzi, K. N. \& Powell, J. D. Regulation of T cells by mTOR: the known knowns and the known unknowns. Trends Immunol. 36, 13-20 (2015).

50. Walmsley, S. R. et al. Hypoxia-induced neutrophil survival is mediated by HIF-1alpha-dependent NF-kappaB activity. J. Exp. Med. 201, 105-115 (2005).

51. Jaakkola, P. et al. Targeting of HIF-alpha to the von Hippel-Lindau ubiquitylation complex by O2-regulated prolyl hydroxylation. Science 292, 468-472 (2001).

52. Suzuki, S., Toledo-Pereyra, L. H., Rodriguez, F. J. \& Cejalvo, D. Neutrophil infiltration as an important factor in liver ischemia and reperfusion injury. Modulating effects of FK506 and cyclosporine. Transplantation 55, 1265-1272 (1993).

53. Ke, B. et al. KEAP1-NRF2 complex in ischemia-induced hepatocellular damage of mouse liver transplants. J. Hepatol. 59, 1200-1207 (2013).

54. Ke, B. et al. Adoptive transfer of ex vivo HO-1 modified bone marrow-derived macrophages prevents liver ischemia and reperfusion injury. Mol. Ther. $\mathbf{1 8}$ 1019-1025 (2010).

55. Yue, S. et al. Prolonged ischemia triggers necrotic depletion of tissue-resident macrophages to facilitate inflammatory immune activation in liver ischemia reperfusion injury. J. Immunol. 198, 3588-3595 (2017). 